> La pharmacogénétique de la leucémie lymphoblastique aiguë (LLA) a connu une évolution rapide ces dernières années. Les découvertes récentes concernent les composants clés du traitement des LLA : le 6-mercaptopurine et le méthotrexate. La leucémie est le cancer le plus fréquent chez l'enfant, la LLA constituant $80 \%$ de tous les cas de leucémies. Les protocoles de traitement incluant plusieurs agents chimiothérapeutiques améliorent nettement les taux de survie chez ces patients. Néanmoins, la LLA reste la cause principale de mortalité chez l'enfant cancéreux. Les différences interindividuelles de réponse aux médicaments représentent une cause importante de résistance et de toxicité au traitement. L'identification des déterminants pharmacogénétiques des médicaments utilisés pour le traitement de la LLA permettrait de repérer les patients répondant de façon sous optimale, et de constituer un complément aux protocoles traditionnels en ajustant la dose de médicaments selon le génotype. <

\section{too añ \\ a taire grandir la vie.}

\title{
Pharmacogénétique de la leucémie lymphoblastique aiguë
}

Marc Ansari, Geneviève St-Onge, Maja Krajinovic

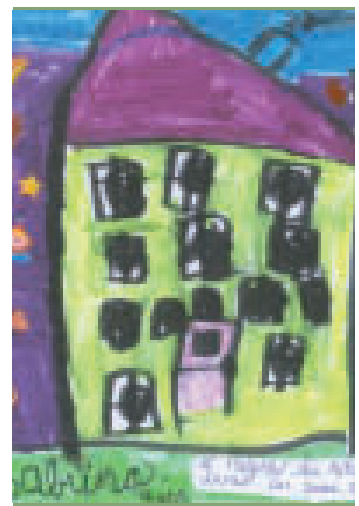

tion d'activité de cette enzyme est causée, à $95 \%$, par trois allèles: TPMT 2 (G238C), TPMT $3 A$ (G460A et $A 719 G)$ et TPMT 3C (A719G) (Tableau 1) [1]. Une activité basse ou nulle de TPMT peut provoquer, à un dosage conventionnel, une myélosuppression sévère, ce qui exige une réduction de la dose pour éviter la toxicité. Dans certaines institutions, le génotype de TPMT est déterminé chez les patients avec LLA avant l'administration de 6-mercaptopurine (6-MP) et les doses sont ajustées en fonction du génotype. La Food and Drug Administration vient d'accepter le génotypage du TPMT afin de diminuer le taux de toxicité sévère [2].

Le taux de survie aux cancers pédiatriques a augmenté de $10 \%$ à $70 \%$ en moins de 50 ans. Néanmoins, beaucoup d'enfants rechutent encore ou souffrent des toxicités des traitements: la résistance aux médicaments constitue la cause principale de mortalité chez l'enfant cancéreux. La réponse individuelle à un médicament s'explique par une interaction complexe de facteurs génétiques et d'autres facteurs. La pharmacogénétique cherche à identifier des variations de l'ADN qui pourraient prédire la réponse des patients au traitement.

\section{Polymorphismes génétiques et thiopurines}

La thiopurine S-méthyltransférase (TPMT) est une enzyme qui métabolise les thiopurines. La réduc-

\section{Pharmacogénétique du méthotrexate (MTX)}

L'efficacité du MTX a été démontrée dans le traitement de la LLA chez l'enfant (Figure 2) [3]. Le MTX est un antagoniste du folate qui inhibe l'enzyme dihydrofolate réductase (DHFR), empêchant la synthèse de tétrahydrofolate (THF) à partir du dihydrofolate (DHF) et menant à un manque des coenzymes du folate (Figure 1). Sous sa forme polyglutamate, le MTX inhibe de façon directe des enzymes participant à la synthèse des purines et des pyrimidines. Les niveaux de folates sont maintenus entre autres par la 5,10-méthylènetétrahydrofolate réductase (MTHFR), qui 
convertit le 5,10-méthylènetétrahydrofolate (5,10-méthylène-THF) en 5-méthyltétrahydrofolate (5-méthyl-THF). Le 5,10-méthylène-THF est un cofacteur de la thymidylate synthase (TS), une enzyme clé de la synthèse nucléotidique qui catalyse la conversion du dUMP en dTMP.

Les mécanismes les plus fréquemment en cause dans la résistance au MTX sont une modification de l'expression des enzymes cibles, une diminution de la synthèse des MTX polyglutamates (MTXPG) influencée par l'action de la folylpolyglutamate synthase (FPGS) et de la $\gamma$-glutamyl hydrolase (GGH), et/ou une baisse de l'apport intracellulaire du MTX résultant d'une activité diminuée des transporteurs des folates réduits (RFCl) [3].

\begin{tabular}{|c|c|c|c|c|c|c|c|c|}
\hline Gène & $\begin{array}{l}\text { Poly- } \\
\text { morphisme }\end{array}$ & Localisation & $\begin{array}{c}\text { Fréquence } \\
\%\end{array}$ & Fonction & $\begin{array}{l}\text { Nombre } \\
\text { de } \\
\text { patients }\end{array}$ & Médicament & Protocole & Références \\
\hline \multirow[t]{3}{*}{ TPMT } & G238C & Exon 5 & 0.2 & + & 182 & 6-MP & St-Jude & {$[27,28]$} \\
\hline & G460A & Exon 7 & 3 & & & & & \\
\hline & A719G & Exon 10 & 3 & & & & & \\
\hline \multirow[t]{4}{*}{ TS } & $2 R / 3 R$ & 5' UTR & $50-60$ & + & 200 & MTX & $\mathrm{DFCl}$ & [10] \\
\hline & & & & & 247 & & St-Jude & [13] \\
\hline & & & & & 64 & & St-Jude & [12] \\
\hline & $\begin{array}{l}2 R / 3 R \\
/ / 6 b p-/ 6 b p+\end{array}$ & 3' UTR & 29 & & 259 & & $\mathrm{DFCl}$ & [11] \\
\hline \multirow[t]{3}{*}{ MTHFR } & $\mathrm{C} 677 \mathrm{~T}$ & Exon 5 & 35 & + & 201 & MTX & $\mathrm{DFCl}$ & [4] \\
\hline & & & & & 520 & & CCG & {$[5]$} \\
\hline & & & & & 186 & & $\mathrm{DFCl}$ & [6] \\
\hline \multirow[t]{2}{*}{ CCNDI } & A870G & Exon/Intron 4 & 58 & + & 205 & MTX & $\mathrm{DFCl}$ & [16] \\
\hline & & & & & 183 & & inconnu & [29] \\
\hline GR & $\mathrm{Bcll}$ & Intron & 29 & $+\star$ & 222 & CS & $\mathrm{DFCl}$ & [17] \\
\hline \multirow{4}{*}{ GST } & $\begin{array}{l}\text { GSTTl/GSTTl } \\
\text { (délétion) }\end{array}$ & & 20 & & 60 & Agents alkylants, & BFM & [20] \\
\hline & & Gène & & + & 135 & anthracycline, & BFM & [30] \\
\hline & GSTMI/GSTMI & & 50 & & & CS & & \\
\hline & (délétion) & & 50 & & 246 & & St-Jude & [12] \\
\hline \multirow[t]{2}{*}{ cyp } & СуP $1 A 1 * 2 A$ & & & + & 320 & CS & $\mathrm{DFCl}$ & [22] \\
\hline & (T6235C) & 3' UTR & 19 & & & & & \\
\hline \multirow[t]{2}{*}{ MDRl } & C3435T & & & + & 111 & Anthracycline, & BFM & [23] \\
\hline & & Exon 26 & 46 & & & $\begin{array}{l}\text { etoposide, } \\
\text { cyclophospha- } \\
\text { mide, CS }\end{array}$ & & \\
\hline
\end{tabular}

Tableau I. Polymorphismes ayant un impact positif sur la réponse thérapeutique de la leucémie lymphoblastique aiguë de l'enfant. CCG : Children's Cancer Study Group ; DFCI : Dana-Farber Cancer Institute ; BFM : Berlin-Frankfurt-Münster Group ; TPMT : thiopurine méthyltransférase ; TS : thymidylate synthase ; MTHFR : méthylène tétrahydrofolate réductase ; CCNDl : cycline Dl ; GR : récepteur des glucocorticoïdes ; CS : corticostéroïdes ; MTX : méthotrexate ; 6-MP : 6-mercaptopurine ; GST : glutathion S-transférase ; CYP : cytochrome ; MDR1 : multidrug resistance. *Pour le gène GR, aucun rôle fonctionnel direct du variant Bcll n'a été démontré; cependant, ce polymorphisme est associé à une diminution de la sensibilité aux corticostéroïdes. 


\section{5,10-méthylène tétrahydrofolate réductase (MTHFR)}

Des niveaux réduits de 5 -méthyl-THF peuvent entraîner une diminution de la méthylation de l'homocystéine en méthionine, menant à une hypercystéinémie et à une hypométhylation de l'ADN. En revanche, de bas niveaux de 5,10-méthylène-THF, requis pour la synthèse de thymidylate, peuvent mener à l'incorporation d'uracile dans I'ADN, entraînant une hausse de la fréquence des dommages chromosomiques, ce qui facilite l'action de certains agents chimiothérapeutiques tel le MTX [3]. Deux polymorphismes dans le gène MTHFR, C677T et A1298C mènent à des remplacements d'acides aminés et à une activité enzymatique réduite (Tableau I). L'augmentation résultante des niveaux de 5,10méthylène-THF peut faciliter la synthèse de thymidylate et ainsi antagoniser l'action du MTX. À l'Hôpital Ste-Justine, 201 enfants atteints de LLA recevant du MTX selon les protocoles de traitement du Dana-Farber Cancer Institute (DFCl) ont été analysés pour les polymorphismes du MTHFR [4]. Un risque augmenté de rechute a été observé chez les patients ayant au moins un allèle MTHFR T677, observation confirmée dans une cohorte de 520 patients [5]. L'analyse de la relation entre les génotypes de MTHFR et la fréquence des toxicités hématologique et hépatique indique un niveau plus bas de leucopénies de grade 3 chez les patients atteints de LLA ayant I'allèle T677 [6]. Cela suggère que ce variant confère une sensibilité réduite au traitement. D'autres études ont également fait état d'une absence d'association entre les polymorphismes de MTHFR et différents types de toxicité examinés $[4,5]$. Les patients ayant des génotypes associés à une survie sans événement diminuée et une absence de toxicité ou développant une toxicité moins fréquente pourraient bénéficier d'une dose augmentée de médicaments. L'identification systématique des polymorphismes et des haplotypes communs dans le gène MTHFR suivie d'études génomiques fonctionnelles vient récemment d'être rapportée [7]. Ceci permettra probablement une meilleure compréhension des polymorphismes de MTHFR dans la réponse thérapeutique des patients atteints de LLA.

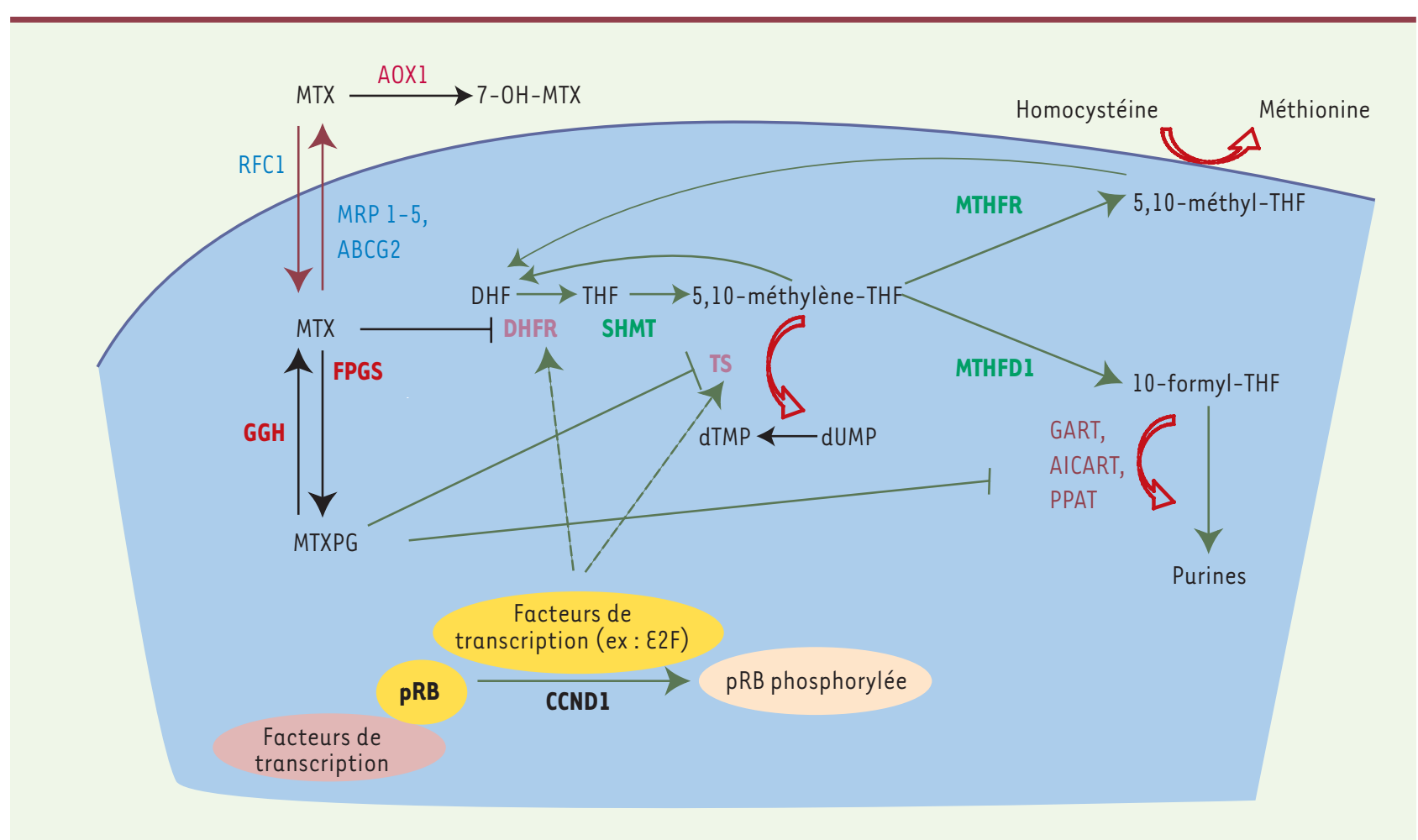

Figure 1. Action du MTX et son interférence avec le cycle des folates. RFCl : transporteur des folates réduits; MRP 1-5: famille des protéines associées aux résistances thérapeutiques multiples; $A B C G 2$ : membre de la famille des transporteurs $A B C$ également connu comme la protéine de résistance au cancer du sein (breast cancer resistance protein, BCRP); MTXPG : MTX polyglutamates; FPGS : folylpolyglutamate synthase; GGH : $\gamma$-glutamyl hydrolase; AOXI : aldéhyde oxydase; DHF : dihydrofolate; THF : tétrahydrofolate; DHFR : dihydrofolate réductase ; TS : thymidylate synthase; dUMP : désoxyuridine monophosphate; dTMP : désoxythymidine monophosphate; GART : glycénamide ribonucléotide transformylase; AICART : aminoimidazole carboxamide ribonucléotide transformylase; PPAT : amido phosphoribosyltransférase ; MTHFR : méthylène-tétrahydrofolate réductase; MTHFDI : méthylène-tétrahydrofolate déhydrogénase; SHMT : sérine hydroxyméthyltransférase; pRB : protéine du rétinoblastome ; CCNDl : cycline Dl ; ع2F : facteur de transcription. Les transporteurs du MTX sont indiqués en bleu, les enzymes ciblées par le MTX sont en violet, les enzymes impliquées dans le métabolisme du MTX sont en rouge, et celles impliquées dans la synthèse de facteurs du cycle des folates sont en vert. 


\section{Thymidylate synthase (TS)}

Plusieurs polymorphismes du gène TS correspondant à des niveaux d'ARNm ou d'expression enzymatique variables ont été décrits: une séquence répétée double $(2 R)$ ou triple $(3 R)$ de 28 paires de base $(\mathrm{pb})$ dans l'élément activateur de la région 5'UTR du gène,

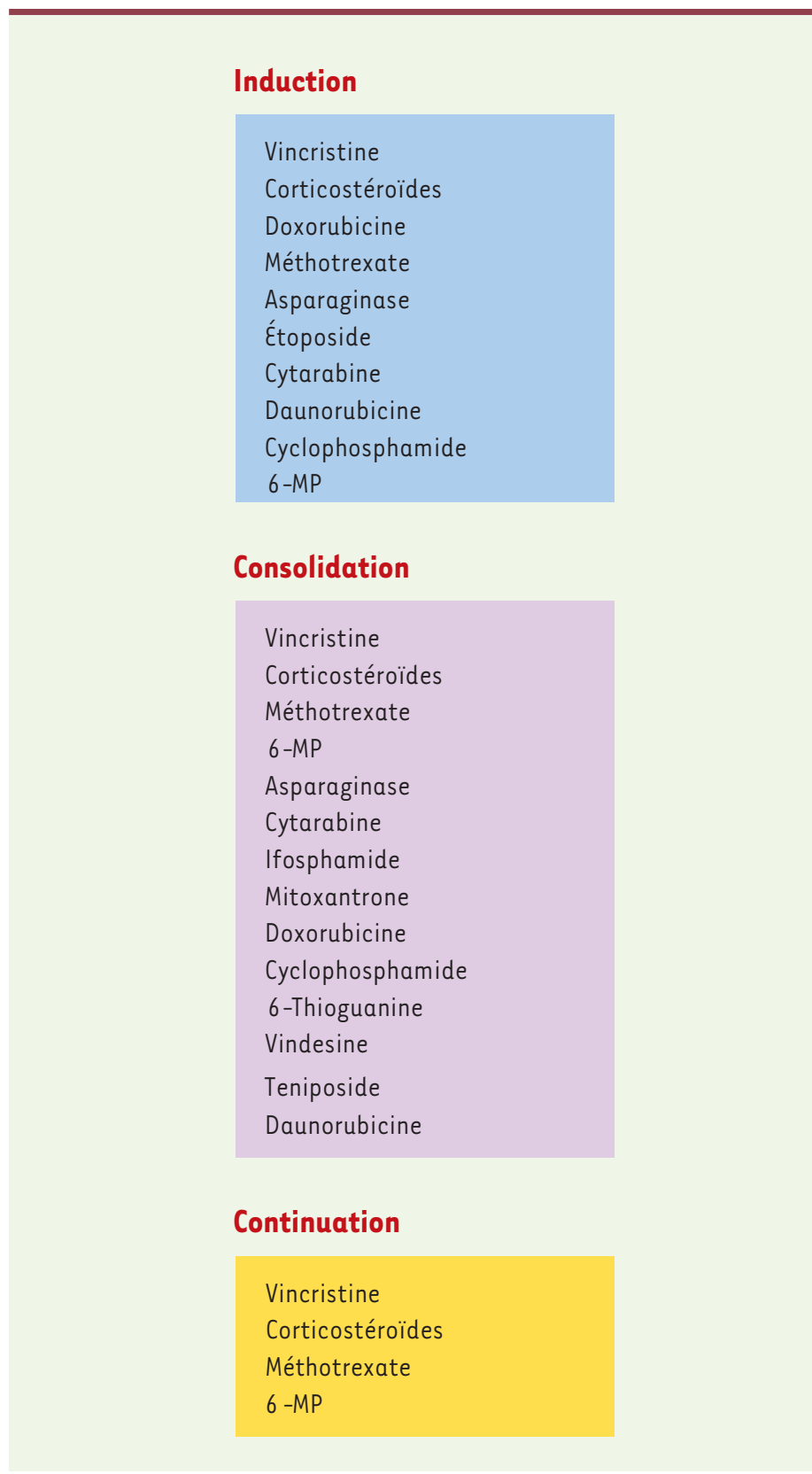

Figure 2. Types de médicaments utilisés dans le traitement de la LLA. Représentation générale de certains protocoles utilisés dans le traitement de la LLA. II n'y a aucune mention ici du traitement préventif du système nerveux central. Le traitement diffère en fonction du groupe de risque (haut risque, intermédiaire, risque standard). Les protocoles auxquels il est fait référence dans cette figure sont ceux du DanaFarber Cancer Institute (DFCI), du St.Jude's Hospital (Protocole Total XIIIB), du Children Cancer Group (CCG-1891) et du Berlin-Frankfurt-Münster Group (BFM). une substitution de $G$ à $C$ de l'allèle $3 R$ et une insertion/délétion de $6 \mathrm{pb}$ dans la région 3'UTR [8,9]. Les séquences répétées semblent fonctionner comme élément activateur, des études in vitro ont montré que l'allèle $3 R$, comparativement à l'allèle $2 R$, augmente l'expression et l'efficacité de la traduction de TS [8]. Il a été démontré que des patients homozygotes pour l'allèle $3 R$ ont un taux de survie sans événement plus faible en comparaison avec les patients sans ce génotype (Tableau I) [10]. Les autres variants $T S$, sans qu'ils n'influencent individuellement les probabilités de survie sans événement chez les patients atteints de LLA, semblent potentialiser ou antagoniser l'effet de l'allèle $3 R$. À ce titre, le fait d'être homozygote pour la délétion de $6 \mathrm{pb}$ semble atténuer l'effet du variant $3 R$ [11]. Une autre étude a établi que le génotype $3 R 3 R$ prédispose les patients à bas risque à une rechute au niveau du système nerveux central [12]. À noter que les patients avec une activité enzymatique réduite $(2 R 2 R)$, conférant une sensibilité accrue au MTX, présentent une incidence plus élevée d'ostéonécrose de la hanche [13].

\section{Autres gènes candidats importants pour la pharmacogénétique du MTX} L'enzyme GGH retransforme le MTXPG en MTX, ce qui pourrait causer un efflux plus élevé et donc conduire à une diminution de l'efficacité du médicament (Figure 1). Dans le promoteur du gène GGH, plusieurs polymorphismes modifiant l'expression du gène ont été décrits [14]. La substitution C452T affecte l'accumulation de MTXPG. Toutefois, des résultats suggèrent une contribution relativement mineure des variants de GGH aux réponses thérapeutiques dans la LLA chez l'enfant [15].

La cycline Dl (CCNDl) est une protéine qui participe à la régulation de la phase $\mathrm{Gl}$ du cycle cellulaire. Elle joue un rôle dans la phosphorylation de la protéine pRB (Figure 1 et Tableau I). Cette phosphorylation de $p R B$ peut être affectée par une expression accrue de CCNDl. II en résulte des niveaux plus élevés du facteur de transcription $\varepsilon 2 F$, ce qui peut mener à une transcription augmentée des cibles du MTX, telles DHFR et TS, et potentiellement affecter la sensibilité au MTX. II a été montré que le polymorphisme CCNDI A870G modifie l'épissage de l'ARNm. Les patients LLA homozygotes pour le variant CCNDI A870 doivent compter sur un moins bon pronostic, une fréquence diminuée des semaines de toxicité hématologique et hépatique [6] et, moins fréquemment, une dose réduite de MTX, en raison de la toxicité [16]. 


\section{Interactions gène/gène}

Les MTXPG inhibent directement l'enzyme TS; or l'activité de TS requiert la présence de 5,10-méthylène-THF ; il est alors possible que les effets des gènes régulant l'activité de TS et les niveaux des cofacteurs folates aient des répercussions plus grandes lorsqu'ils sont combinés. De plus bas niveaux de TS et de 5,10-méthylène-THF faciliteraient l'action du MTX, tandis que des niveaux plus élevés mèneraient à une résistance à ce médicament. On s'attendait à ce phénomène chez des individus homozygotes pour le variant TS $3 R$ dotés aussi des variants de MTHFR qui court-circuiteraient le métabolisme des folates en vue de la conservation du 5,10-méthylène-THF (Figure 1) [3]. II a d'ailleurs été démontré que les enfants atteints de LLA porteurs à la fois des haplotypes TS 3R3R et MTHFR T677A1298 comptent des probabilités fortement réduites de survie sans événement, comparativement aux patients n'ayant pas ces génotypes [4].

Une modification de la régulation de pRB par l'action de CCNDl peut entraîner une expression accrue des cibles du MTX, menant ainsi à une baisse de la sensibilité au MTX. L'analyse des effets combinés des polymorphismes pour CCNDl et TS chez des enfants atteints de LLA démontre que les individus porteurs des génotypes CCNDI AA870 et $T S 3 R 3 R$ ont une probabilité réduite de survie sans événement, comparativement aux individus n'ayant pas de génotype prédisposant à l'événement [16].

D'autres polymorphismes s'accompagnent aussi d'un effet accru lorsqu'ils sont combinés à l'action des variants de TS. Dans leur étude, Rocha et al. ont montré que les patients du groupe à haut risque ayant le génotype non-nul pour le gène de la glutathione S-transférase (GSTMI) étaient exposés à un risque augmenté de rechute hématologique, risque encore plus élevé en présence du génotype TS $3 R 3 R$ [12].

\section{Pharmacogénétique des corticostéroïdes (CS)}

Les CS font partie des protocoles de traitement des LLA (Figure 2) [1] ; la sensibilité aux CS est un facteur de pronostic important. Les CS peuvent jouer leur rôle thérapeutique en se liant d'abord au récepteur intracellulaire des glucocorticoïdes pour réguler ensuite la transcription des gènes en aval. Une étude du DFCl sur le récepteur des glucocorticoïdes montre une réduction de survie globale chez les enfants LLA homozygotes pour l'allèle $G$ du polymorphisme $B c l l$, association particulièrement évidente chez les patients présentant un risque élevé (Tableau I) [17]. En revanche, le Dutch Childhood Oncology Group, dans une étude sur 57 patients traités pour la LLA, n'observe aucune association entre ce polymorphisme et une résistance in vivo ou in vitro aux glucocorticoïdes [18]. Des différences liées à la conception des études, au nombre de patients considérés et au protocole de traitement pourraient expliquer ces divergences de conclusion. Le variant $B c l l$ étant un polymorphisme intronique, la possibilité d'un déséquilibre de liaison (association non aléatoire d'allèles à deux ou plusieurs locus) avec d'autres variants non encore identifiés ne peut être exclue. Ces informations s'ajoutent à celles qui avaient démontré que les polymorphismes du gène de la glutathione-S-transférase (GST) jouent un rôle dans la réponse aux glucocorticoïdes [19].

\section{Autres gènes candidats importants dans la pharmacogénétique de la LLA}

Plusieurs médicaments sont utilisés dans le traitement de Ia LLA (Figure 2) et différents gènes exercent un rôle dans leur métabolisme. La GST est partie prenante dans le métabolisme et la détoxification de plusieurs agents utilisés dans le traitement de la LLA. Une délétion homozygote des gènes GSTMI et GSTTI mène à une activité réduite ou nulle de l'enzyme, ce qui peut diminuer sa fonction dans la métabolisation des agents chimiothérapeutiques. Stanulla et al. [20] ont démontré que le génotype GSTTl null protégeait les patients contre une rechute. Au St-Jude's Hospital, une étude concernant les enfants du groupe haut risque traités selon le protocole total XIIIB, a souligné que ceux ayant une ou deux copies de GSTMI comptaient un risque plus grand de rechute (Tableau 1) [12]. Ce constat n'a cependant pas été confirmé dans d'autres études qui n'ont pas considéré l'effet du polymorphisme après la stratification en groupe de risque. [21, 22].

Les cytochromes comptent beaucoup dans le métabolisme de nombreux médicaments chimiothérapeutiques (Figure 2). À l'Hôpital Ste-Justine, on a observé que la survie sans événement des patients porteurs du variant $C Y P I A I * 2 A$, caractérisé par l'allèle $C$ du polymorphisme T6235C, est réduite (Tableau I) [22].

Les multidrug resistance proteins (MDR) forment une famille de transporteurs bien identifiés pour le rôle qu'ils exercent dans I'efflux de médicaments importants dans le traitement de la LLA (Figure 2). II a été démontré, chez les enfants traités selon le protocole BFM, une diminution de la survie sans événement des homozygotes pour l'allèle C3435 du gène MDRI (Tableau I) [23].

Enfin, il convient de noter que parmi les agents chimiothérapeutiques prescrits dans le traitement de la LLA (Figure 2), I'asparaginase n'a pas encore été étudiée du point de vue de la pharmacogénétique. Ce médicament est important dans le traitement de la LLA : il dégrade l'asparagine, acide aminé essentiel à la survie de la cellule cancéreuse.

\section{Conclusion}

Le transfert clinique des connaissances acquises en pharmacogénomique pourrait rendre plus efficaces les médicaments chimiothérapeutiques en individualisant leur sélection ainsi que 
leur dose et, de ce fait, améliorer le traitement du cancer [24]. Il a été démontré que l'efficacité d'un médicament est augmentée et sa toxicité diminuée lorsque la stratégie d'ajustement de la dose est guidée par la génétique [25]. À l'heure actuelle, le génotypage du TPMT a été incorporé au traitement de la leucémie. Profitable pour le patient, il s'accompagne en outre d'un rapport coût-bénéfice avantageux $[25,26]$.

Néanmoins, comme les résultats de certaines études sont controversés, le besoin de mieux comprendre l'association entre les facteurs génétiques et le traitement demeure encore nécessaire. II apparaît difficile de comparer des études fondées sur différents protocoles de traitement, et qui, de plus, considèrent parfois l'utilisation de médicaments différents (Figure 2) et de dosages différents. L'effet des facteurs génétiques pourrait être modulé et mieux appréhendé selon les paramètres de stratification intra-protocole (âge, sexe, groupes de populations, caractéristiques de la maladie, classes de risque, etc.). De plus, la majorité des études ne sont pas prospectives et les patients recrutés ne sont pas forcément comparables; le recrutement des patients a pu avoir été fait à des moments où d'autres facteurs auraient pu jouer un rôle (habitudes alimentaires et facteurs environnementaux variables). Plusieurs études n'analysent qu'un gène à la fois, d'autres n'utilisent les données que d'un petit nombre de patients, ces conditions augmentent le risque de fausses associations, d'autant plus qu'un ajustement pour les tests multiples n'est souvent pas fait. Des études prospectives bien construites, avec des cohortes de grande taille, sont donc nécessaires afin de confirmer certains résultats. Une approche reposant sur l'haplotype (groupe de polymorphismes, sur un même chromosome, qui sont statistiquement associés) plutôt que sur le génotype, et qui intégrerait une interaction gène/gène plutôt que l'action d'un seul gène, pourrait fournir une estimation plus complète et plus utile des différences génétiques individuelles. $\diamond$

\section{REMERCIEMENTS}

Le travail des auteurs est possible grâce au soutien de l'Institut canadien de la recherche en cancer, le Fonds de recherche canadien de la leucémie, le Centre de recherche du CHU SainteJustine, le Centre d'excellence en Oncologie pédiatrique et soins palliatifs et la Fondation Charles-Bruneau. Maja Krajinovic est bénéficiaire du Fonds de la recherche en santé du Québec et Marc Ansari est soutenu par la Fondation Charles-Bruneau et le Fonds national Suisse de la recherche.

\section{SUMMARY}

Pharmacogenomics of acute lymphoblastic leukemia

Pharmacogenomics of acute lymphoblastic leukemia ( $A L L$ ) evolved rapidly in the past few years. Majority of recent findings concerns knowledge on key components of ALL treatment, 6-mercaptopurine and methotrexate. Leukemia is the most common cancer affecting children, with ALL comprising $80 \%$ of all leukemia cases. Introduction of treatment protocols composed of several chemotherapeutic agents improved importantly survival in patients with ALL. Nevertheless, ALL is still the leading cause of cancer-related death in children. Interindividual differences in drug responses are an important cause of resistance to treatment and adverse drug reactions. Identifying pharmacogenomic determinants of drugs used in ALL treatment may allow for prospective identification of patients with suboptimal drug responses allowing for complementation of traditional treatment protocols by genotype-based drug dose adjustment. $\diamond$

\section{GLOSSAIRE}

6-MP : 6-mercaptopurine

CCND1 : cycline D1

CS : corticostéroïdes

DFCI : Dana-Farber Cancer Institute

GGH : gamma glutamate hydrolase

GST : glutathion S-transférase

GR : récepteur des glucocorticoïdes (glucocorticoid receptor)

Hcy : homocystéine

LLA : leucémie lymphoblastique aiguë

MRP : protéines associées aux résistances thérapeutiques multiples (multidrug resistant proteins)

MTHFR : 5,10-méthylènetétrahydrofolate réductase

MTRR : méthionine synthase réductase

MTX : méthotrexate

MTXPG : MTX polyglutamates

RFCl : transporteur des folates réduits (reduced folate carrier)

SHMT1 : sérine hydroxyméthyl transférase

THF : tétrahydrofolate

TPMT : thiopurine S-méthyltransférase

TS: thymidylate synthase

\section{RÉFÉRENCES}

1. Cheok MH, Lugthart S, Evans WE. Pharmacogenomics of acute leukemia. Annu Rev Pharmacol Toxicol $2006 ; 46: 317-53$.

2. Maitland ML, Vasisht K, Ratain MJ. TPMT, UGTIAl and DPYD : genotyping to ensure safer cancer therapy? Trends Pharmacol Sci $2006 ; 27: 432-7$.

3. Krajinovic M, Moghrabi A. Pharmacogenetics of methotrexate. Pharmacogenomics 2004 ; $5: 819-34$.

4. Krajinovic M, Lemieux-Blanchard $\varepsilon$, Chiasson S, et al. Role of polymorphisms in MTHFR and MTHFDl genes in the outcome of childhood acute lymphoblastic leukemia. Pharmacogenomics J $2004 ; 4: 66-72$.

5. Aplenc R, Thompson J, Han P, et al. Methylenetetrahydrofolate reductase polymorphisms and therapy response in pediatric acute lymphoblastic leukemia. Cancer Res $2005 ; 65: 2482-7$. 
6. Costea I, Moghrabi A, Laverdiere C, et al. Folate cycle gene variants and chemotherapy toxicity in pediatric patients with acute lymphoblastic leukemia. Haematologica $2006 ; 91: 1113-6$.

7. Martin $\mathrm{YN}$, Salavaggione $0 \varepsilon$, Eckloff BW, et al. Human methylenetetrahydrofolate reductase pharmacogenomics : gene resequencing and functional genomics. Pharmacogenet Genomics 2006; $16: 265-77$.

8. Horie $\mathrm{N}$, Aiba H, Oguro K, et al. Functional analysis and DNA polymorphism of the tandemly repeated sequences in the 5'-terminal regulatory region of the human gene for thymidylate synthase. Cell Struct Funct $1995 ; 20: 191-7$.

9. Pullmann R Jr, Abdelmohsen K, Lal A, et al. Differential stability of thymidylate synthase 3 '-untranslated region polymorphic variants regulated by AUFl. J Biol Chem 2006; $281: 23456-63$.

10. Krajinovic M, Costea I, Chiasson S. Polymorphism of the thymidylate synthase gene and outcome of acute lymphoblastic leukaemia. Lancet $2002 ; 359$ : 1033-4.

11. Krajinovic M, Costea I, Primeau M, et al. Combining several polymorphisms of thymidylate synthase gene for pharmacogenetic analysis. Pharmacogenomics / $2005 ; 5: 374-80$

12. Rocha JC, Cheng C, Liu W, et al. Pharmacogenetics of outcome in children with acute lymphoblastic leukemia. Blood $2005 ; 105$ : 4752-8.

13. Relling MV, Yang W, Das S, et al. Pharmacogenetic risk factors for osteonecrosis of the hip among children with leukemia. J Clin Oncol $2004 ; 22: 3930-6$

14. Chave KJ, Ryan TJ, Chmura SE, Galivan J. Identification of single nucleotide polymorphisms in the human gamma-glutamyl hydrolase gene and characterization of promoter polymorphisms. Gene 2003 ; 319: 167-75.

15. Garcia-Bournissen F, Moghrabi A, Krajinovic M. Therapeutic responses in childhood acute lymphoblastic leukemia (ALL) and haplotypes of gamma glutamyl hydrolase (GGH) gene. Leuk Res 2007 ; 31 : 1023-5.

16. Costea I, Moghrabi A, Krajinovic M. The influence of cyclin Dl (CCNDI) 870A-G polymorphism and CCNDl-thymidylate synthase (TS) gene-gene interaction on the outcome of childhood acute lymphoblastic leukaemia. Pharmacogenetics $2003 ; 13: 577-80$.

17. Fleury I, Primeau M, Doreau A, et al. Polymorphisms in genes involved in the corticosteroid response and the outcome of childhood acute lymphoblastic leukemia. Am J Pharmacogenomics 2004 ; 4 : 331-41.

18. Tissing WJ, Meijerink JP, den Boer ML, et al. Genetic variations in the glucocorticoid receptor gene are not related to glucocorticoid resistance in childhood acute lymphoblastic leukemia. Clin Cancer Res 2005 ; $11: 6050-6$
19. Meissner B, Stanulla M, Ludwig WD, et al. The GSTTl deletion polymorphism is associated with initial response to glucocorticoids in childhood acute lymphoblastic leukemia. Leukemia $2004 ; 18: 1920-3$.

20. Stanulla M, Schrappe M, Brechlin AM, et al. Polymorphisms within glutathione S-transferase genes (GSTMI, GSTTI, GSTP1) and risk of relapse in childhood B-cell precursor acute lymphoblastic leukemia: a case-control study. Blood $2000 ; 95$ : 1222-8.

21. Davies SM, Bhatia S, Ross JA, et al. Glutathione S-transferase genotypes, genetic susceptibility, and outcome of therapy in childhood acute lymphoblastic leukemia. Blood $2002 ; 100: 67-71$.

22. Krajinovic $M$, Labuda $D$, Mathonnet $G$, et al. Polymorphisms in genes encoding drugs and xenobiotic metabolizing enzymes, DNA repair enzymes, and response to treatment of childhood acute lymphoblastic leukemia. Clin Cancer Res $2002 ; 8: 802-10$.

23. Jamroziak K, Mlynarski W, Balcerczak $\varepsilon$, et al. Functional C3435T polymorphism of MDRl gene : an impact on genetic susceptibility and clinical outcome of childhood acute lymphoblastic leukemia. Eur J Haematol $2004 ; 72: 314-21$.

24. Ansari M, Krajinovic M. Pharmacogenomics in cancer treatment defining genetic bases for inter-individual differences in responses to chemotherapy. Curr Opin Pediatr 2007 $19: 15-22$

25. Guo Y, Shafer $S$, Weller $P$, et al. Pharmacogenomics and drug development. Pharmacogenomics $2005 ; 6: 857-64$

26. Van den Akker-van Marle ME, Gurwitz D, Detmar SB, et al. Cost-effectiveness of pharmacogenomics in clinical practice: a case study of thiopurine methyltransferase genotyping in acute lymphoblastic leukemia in Europe. Pharmacogenomics $2006 ; 7: 783-92$

27. Relling MV, Pui $\mathrm{CH}$, Cheng $\mathrm{C}$, Evans WE. Thiopurine methyltransferase in acute lymphoblastic leukemia. Blood $2006 ; 107: 843-4$.

28. Relling MV, Hancock ML, Rivera GK, et al.. Mercaptopurine therapy intolerance and heterozygosity at the thiopurine S-methyltransferase gene locus. J Natl Cancer Inst 1999 ; $91: 2001-8$.

29. Hou X, Wang $S$, Zhou $Y$, et al. Cyclin Dl gene polymorphism and susceptibility to childhood acute lymphoblastic leukemia in a Chinese population. Int J Hematol 2005 ; 82 : 206-9.

30. Anderer G, Schrappe M, Brechlin AM, et al. Polymorphisms within glutathione S-transferase genes and initial response to glucocorticoids in childhood acute lymphoblastic leukaemia. Pharmacogenetics $2000 ; 10: 715-26$.

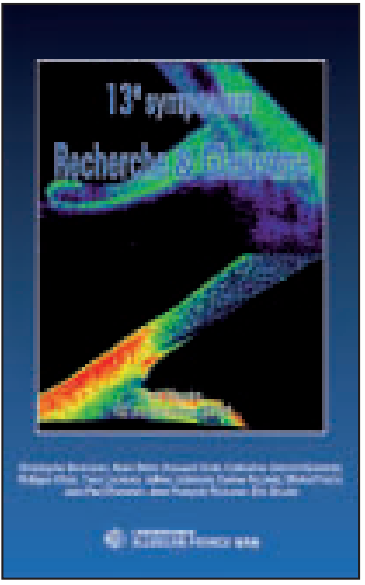

\section{Bon de commande}

À retourner à EDK, 2, rue Troyon - 92316 Sèvres Cedex

Tél. : 0155641393 - Fax : 0155641394 - E-mail : edk@edk.fr

NOM :

Prénom

Adresse :

Code postal :

Ville :

Pays :

Fonction :

Je souhaite recevoir l'ouvrage $13^{\mathrm{e}}$ Symposium Recherche et Glaucome :

$25 €+3 €$ de port $=\mathbf{2 8} €$ TTC

en ................. exemplaire, soit un total de .......................................... $€$

$\square$ Par chèque, à l'ordre de $\mathbf{E} \mathbf{D}$ K

$\square$ Par carte bancaire : $\square$ Visa $\square$ Eurocard/Mastercard

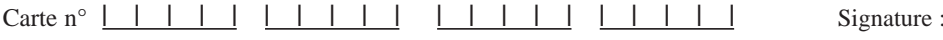

Date d'expiration : $\quad \leq 1|\underline{1}|$

$\mathrm{N}^{\circ}$ de contrôle au dos de la carte :

\section{TIRÉS À PART}

M. Krajinovic 